\title{
CUIDADOS INTEGRALES DE ENFERMERÍA EN UN LACTANTE CON ENCEFALOPATÍA ISQUÉMICA HIPÓXICA RELACIONADA CON LA ASFIXIA PERINATAL
}

\section{COMPREHENSIVE NURSING CARE IN AN INFANT WITH HYPOXIC ISCHEMICAL ENCEPHALOPATHY RELATED TO PERINATAL ASPHYXIA}

\section{CUIDADOS INTEGRAIS DE ENFERMAGEM EM UM LACTANTE COM ENCEFALOPATÍA ISQUÉMICA HIPÓXICA RELACIONADA À ASFIXIA PERINATAL}

\author{
Daniela Sanches Couto \\ Universidad Federal de São Carlos (UFSCar). Beca Capes \\ dsanchescouto@gmail.com \\ ORCID:https://orcid.org/0000-0003-0767-4000
}

\section{Ana Jacqueline Rodriguez Flores \\ Universidad Juárez del Estado de Durango (UJED). \\ ORCID: http://Orcid.org/0000-0002-3057-2507}

\section{Pâmela Roberta de Oliveira}

Universidad Federal de Mato Grosso (UFMT).

ORCID: https://orcid.org/0000-0003-0497-6548

\section{Queli Lisiane Castro Pereira}

Universidad Federal de Mato Grosso (UFMT).

ORCID: https://orcid.org/0000-0001-6965-4887

DOI: $10.22235 /$ ech.v8i2.1847

Recibido: 20/08/2018

Aceptado: 15/05/2019

\section{RESUMEN:}

La encefalopatía isquémica hipóxica, secundaria a la asfixia durante el parto, sigue siendo una importante causa de muerte posnatal y de déficit neurológico permanente en el mundo. Las intervenciones de enfermería siguen siendo el pilar del tratamiento, posibilitando un confort al paciente. El estudio objetivó implementar el proceso de enfermería en el cuidado a un paciente pediátrico con lesión cerebral como consecuencia de la asfixia perinatal. Realizamos un estudio de caso clínico en una Unidad Intensiva Pediátrica en Durango, México, entre noviembre y diciembre de 2017. Los datos fueron obtenidos después de la evaluación, presentados en el proceso de enfermería en sus fases de histórico, evolución, diagnóstico de enfermería, planificación de las intervenciones y resultados obtenidos. Resaltamos la importancia del proceso como foco del trabajo del enfermero, capaz de posibilitar el desarrollo de una asistencia basada en evidencia y también permitir credibilidad en el trabajo de enfermería.

Palabras clave: Diagnóstico de enfermería; Enfermería; Enfermería Pediátrica; Proceso de enfermería. 


\section{SUMMARY:}

Hypoxic ischemic encephalopathy, secondary to asphyxia during childbirth, remains an important cause of postnatal death and permanent neurological deficit in the world. Nursing interventions continue as the pillar of the treatment, allowing some comfort to the patient. The study aimed to implement the nursing process in the care of a pediatric patient with brain damage as a consequence of perinatal asphyxia. We conducted a clinical case study in a Pediatric Intensive Unit in Durango, Mexico, in November and December 2017. The data was obtained after the evaluation and presented in the nursing process in its phases of history, evolution, diagnosis, planning of the interventions and results obtained. We emphasize the importance of the process as a focus of the nurse's work, capable of enabling the development of evidence-based care and also to enhance its credibility.

Keywords: Nursing diagnosis; Nursing; Pediatric Nursing; Nursing process.

\section{RESUMO:}

Encefalopatia isquêmica hipóxica, secundária à asfixia durante o parto, continua sendo uma importante causa de morte pós-natal e déficit neurológico permanente no mundo. As intervenções de enfermagem continuam como pilar do tratamento, permitindo conforto ao paciente. $\mathrm{O}$ estudo objetivou implementar o processo de enfermagem no cuidado ao paciente pediátrico com lesão cerebral decorrente da asfixia perinatal. Realizamos um estudo de caso clínico em uma unidade de terapia pediátrica em Durango, no período de novembro a dezembro de 2017. Os dados foram obtidos após a avaliação, apresentados no processo de enfermagem em suas fases da história, evolução, diagnóstico de enfermagem, planejamento das intervenções e os resultados obtidos. Ressaltamos a importância do processo como foco do trabalho do enfermeiro, capaz de possibilitar o desenvolvimento de cuidados baseados em evidências e também permitir a credibilidade do trabalho da enfermagem.

Palabras-chave: Diagnóstico de enfermagem; Enfermagem; Enfermagem Pediátrica; Processo de enfermagem.

\section{INTRODUCCIÓN}

La encefalopatía hipóxico isquémica (EHI) es consecuencia de la asfixia durante el parto y responsable de generar incapacidades neurológicas (1). El síndrome EHI es un disturbio neurológico que consiste en un proceso dinámico que afecta a recién nacidos a término y pretérmino. Esta lesión cerebral ocurre en dos etapas: en el primer momento la asfixia causa la reducción del flujo sanguíneo hacia el cerebro provocando la primera lesión. La contención sanguínea y de oxígeno en el momento del nacimiento es capaz de acarrear daños en las células del sistema nervioso central, incluso la muerte celular. Segundo, cuando se restaura el flujo sanguíneo en un cerebro lesionado se desencadenan diversos efectos colaterales, pudiendo dañar las células neurales o hasta llevar a la muerte cerebral $(2-4)$.

Según la Organización Mundial de la Salud (OMS), la asfixia perinatal (AP) está entre las tres principales causas de muerte entre los neonatos (5). Hasta el $25 \%$ de los recién nacidos y el $8 \%$ de las muertes de niños menores de 5 años están directamente asociadas a la AP (2). Cada año, de cada 1000 nacidos vivos, tres a cinco presentará AP en el momento del nacimiento, aumentando los riesgos de las secuelas neurológicas (3). De los recién nacidos 
vivos que sufrieron asfixia en el momento del parto, de $15 \%$ al $20 \%$ van a la muerte aún en el período neonatal; de los sobrevivientes, el 25\% manifiesta incapacidad neurológica permanente. Los perjuicios neurológicos de mayor gravedad se presentan como parálisis cerebral, epilepsia e hiperactividad, entre otros agravamientos. Las condiciones de riesgo asociadas a la EHI pueden desarrollarse durante el embarazo, en el momento del parto o después del nacimiento, destacándose el bajo peso al nacer y la prematuridad $(5,7)$.

La asistencia de enfermería enfocada en garantizar la recuperación de la salud del lactante es desarrollada de manera sistematizada e individualizada. Con una mirada humanizada, la asistencia debe ser extendida hasta los padres y familiares, que presentan altos niveles de estrés y ansiedad como consecuencia de la internación del paciente. La enfermería es capaz de suplir necesidades que van más allá de la asistencia directa a la patología, fortaleciendo la relación y proporcionando el vínculo con los padres para intentar tranquilizarlos (8). El equipo de enfermería tiene el papel fundamental en los cuidados de los lactantes con consecuencias de la AP, porque son responsables por asegurar que el tratamiento será realizado de manera correcta, contribuyendo con la evolución positiva del lactante. En el cotidiano de la asistencia de enfermería, dirigidas a los pacientes pediátricos, el Proceso de Enfermería revela los mejores caminos, posibilitando una asistencia de calidad, ya que hay una planificación de las acciones que priorizan al paciente. De esta forma, el estudio tuvo como objetivo e presentar el Proceso de Enfermería ante el caso clínico del lactante con lesión cerebral como consecuencia de la asfixia perinatal internado en una institución pública.

\section{METODOLOGÍA: CASO CLÍNICO}

Se trata de un reporte de caso clínico que fue desarrollado durante el Servicio Social de Enfermería en la unidad de terapia intensiva pediátrica en un hospital infantil en la ciudad de Durango, México, en noviembre y diciembre de 2017. Es un trabajo de carácter descriptivo a partir de la presentación del histórico, evolución, diagnóstico de enfermería, planificación de las intervenciones y resultados obtenidos.

Datos de identificación y diagnóstico médico: MRJ es un bebé masculino de 3 meses y 28 días al 6 de noviembre del 2017 internado en la Unidad de Terapia Intensiva Pediátrica. El diagnóstico medico es: Hipoxia cerebral global. Hipertensión pulmonar moderada (61 $\mathrm{mm} \mathrm{Hg}$ ). Hipertrofia de ventrículo derecho. Atelectasia pulmonar total derecha remitida. Quemosis. Paro cardiorrespiratorio (dos episodios). Estado epiléptico secundario. Encefalopatía hipóxico isquémica secundaria.

Historia prenatal: La madre, primigesta de 18 años, estudiante, sangre $\mathrm{B}+$, que lleva su control prenatal incompleto, ha consumido ácido fólico, sulfato ferroso, se vacuna contra el tétanos y la influenza. Sin histórico de complicación gestacional. A las 37 semanas de gestación se opta por practicar una cesárea debido a una restricción de crecimiento intrauterino.

Antecedentes patológicos: se encontraba ya internado en unidad de terapia intensiva pediátrica debido a diversos padecimientos, siendo el principal diagnóstico una EHI. El recién nacido pesa 1600 gramos, con una talla de $40 \mathrm{~cm}$, sangre $\mathrm{O}+\mathrm{y}$ APGAR de 7 al minuto y 8 a los cinco minutos. 
Se le traslada a la Unidad de Cuidados Intensivos Neonatales donde se le suministra oxigeno por puntas nasales y se le mantiene en incubadora. A las dos semanas de nacido se le diagnostica enfermedad por reflujo gastroesofágico y retardo en vaciamiento gástrico con etiología desconocida. Una semana después se le corrige el frenillo lingual corto. Pasa al área de Crecimiento y Desarrollo cuando se muestra mejoría y aumento de peso. Totalizando 57 días hospitalizado, a los 57 días de vida se solicita la alta voluntaria, pesando 2170 gramos, con anemia, neumonía intrahospitalaria y un diagnostico probable de síndrome Silver-Russell. El síndrome de Silver Russell, donde la mayoría de los casos es esporádico, tiene una relación genética, es una enfermedad caracterizada por el retraso de crecimiento intrauterino y postnatal y perímetro craneal anormal (9). En la nota del alta se pone de manifiesto que el paciente se encuentra hipoactivo, hiporreactivo, pálido, hidratado, con nulo sostén cefálico, tolerando la alimentación por sonda orogástrica, aún requiriendo aporte de oxígeno por puntas nasales, sibilancia respiratoria, peristaltis presente e hipotrófico.

\section{REPORTE CLÍNICO}

A los 3 meses y 19 días de edad ingresa al servicio de Urgencias debido a broncoaspiración mientras se le alimentaba con sonda orogástrica, habiendo presencia de crisis comicial. Sufre un paro cardiorrespiratorio, y se le practican maniobras de reanimación cardiopulmonar. Se inicia ventilación mecánica al colocarse tubo orotraqueal, y en la madrugada del día siguiente se le coloca un catéter central de inserción periférica en miembro inferior derecho. Es internado en la Unidad de Terapia Intensiva, donde se le diagnostica hipoxia cerebral global que deriva en una encefalopatía hipóxico-isquémica, atelectasia pulmonar total derecha que remite, e hipertensión pulmonar moderada, que presuntivamente causó una hipertrofia ventricular derecha detectada más tarde. A su ingreso cuenta con un peso de 3800 gramos y talla de $50 \mathrm{~cm}$. En su hogar se le estuvo alimentando con un sucedáneo de la leche materna con sonda orogástrica. Se inicia tratamiento con medicamentos analgésicos (acetaminofén), antibióticos (cefotaxima y clindamicina), anticonvulsivantes (levetiracetam, fenobarbital y fenitoína), gluconato de calcio, inhibidor de fosfodiesterasa (sildenafil), antieméticos (metoclopramida), diuréticos (furosemida) y antiulcerosos (ranitidina). Luego se agregaron medicamentos oftálmicos como tratamiento para la conjuntivitis que adquiere en el hospital (hipromelosa y prednisolona). Se le coloca una sonda vesical para llevar un control de líquidos estricto. Se indican cinco soluciones: a) $3 \mathrm{ml}$ de ácido valproico aforados en 24 mililitros de solución fisiológica para pasar $1 \mathrm{ml} / \mathrm{h}$, b) $115 \mathrm{ml}$ de solución glucosada al 10\% más 115 mililitros de solución fisiológica con $9 \mathrm{~m} \mathrm{Eq} \mathrm{de} \mathrm{KCl}$ para $24 \mathrm{~h}$, c) $2 \mathrm{ml}$ de dobutamina más $10 \mathrm{ml}$ de solución fisiológica para pasar $0.8 \mathrm{ml} / \mathrm{h}$, d) $0.6 \mathrm{ml}$ de norepinefrina aforados a $24 \mathrm{ml} \mathrm{de}$ solución glucosada al $5 \%$ para pasar $0.6 \mathrm{ml} / \mathrm{h}$, y e) $3.6 \mathrm{ml}$ de midazolam aforados a $24 \mathrm{ml}$ de solución fisiológica para pasar $1 \mathrm{ml} / \mathrm{h}$. Durante la primera semana de estancia en terapia intensiva sufre de otro paro cardiorrespiratorio y sobrevive. Se realiza valoración ocho días después de ser internado.

\section{VALORACIÓN POR PATRONES FUNCIONALES DE SALUD}

Nutricional/metabólico: Ha bajado de 3800 gramos de peso a 3350 gramos. De acuerdo a la tabla de peso para edad en niños, es un peso menor al adecuado para él. El perímetro abdominal es de 32 centímetros. Se encuentra en ayuno pues se espera que se le realice una traqueostomía, la cual ha sido pospuesta debido a su estado delicado. Tiene una glicemia de 
euglicemia. A partir de lo expuesto se diagnosticó el Riesgo de glicemia inestable relacionado a alteración del estado mental, estado de salud física comprometido (7).

Su cabello es fino y escaso con buena implantación. Los ojos son cafés, con pupilas isocóricas, mióticas y reactivas a la luz. Hay edema palpebral, esclerótica enrojecida, presencia de carnosidad en ojo izquierdo y secreciones abundantes. Así se diagnosticó el deterioro de la integridad tisular relacionado a daños en la superficie ocular, efectos secundarios del tratamiento, factores ambientales, lesiones neurológicas con pérdida de reflejo sensorial, ventilación mecánica manifestado por lesión tisular (conjuntiva) (7).

La mucosa de la nariz se encuentra hidratada, rosada, y hay secreción abundante. En la boca las mucosas están hidratadas, hay sangrado escaso debido al tubo orotraqueal y la sonda orogástrica, y hay presencia de sialorrea. Hay edema periférico, se encuentra multipuncionado, hay eritema y descamación en nuca y periné, lesión por causa del pulsioximetro en dorso del pie derecho. La zona del catéter venoso de inserción periférica se encuentra protegida con un apósito, se le realiza curación de la zona diariamente.

Se le realiza una gasometría y entre los datos de importancia se encuentra la presión de dióxido de carbono con un valor de $45 \mathrm{~mm} \mathrm{Hg}$ y el $\mathrm{pH}$ en 7.58. Las plaquetas tienen un valor de $136 \mathrm{x}(10)^{3}$, el hematocrito se encuentra en $30.9 \%$ y la hemoglobina en $10.2 \mathrm{~g} / \mathrm{dl}$. En los tiempos, el TP es de 15.2 segundos, en el TPT no se da la coagulación, y el fibrinógeno tiene un valor de $197.7 \mathrm{mg} / \mathrm{dl}$.

Eliminación: La eliminación intestinal no está presente. Tiene una sonda vesical que se instaló al momento de su ingreso en terapia intensiva, la diuresis es escasa $(10 \mathrm{ml})$, y aumenta considerablemente después de la administración de diurético $(55 \mathrm{ml})$, es amarilla clara. En un día tiene pérdida de líquido de $467 \mathrm{ml}$ y un ingreso total de $582 \mathrm{ml}$, por lo cual se diagnosticó el Exceso de volumen de líquido relacionado a compromiso de los mecanismos reguladores manifestado por aportes superiores a las pérdidas, hipertensión pulmonar moderada, oliguria y edema (7). Se tiene un balance hídrico positivo.

Actividad/ejercicio: En el estado cardiovascular, tiene una frecuencia cardiaca de 90 latidos por minuto, tensión arterial de 102/64 mm Hg, el llenado capilar es menor a 3 segundos. Levantando el diagnóstico de Riesgo de sangrado relacionado a coagulopatías esenciales (7). En cuanto al estado respiratorio, cuenta con ventilación mecánica con una frecuencia respiratoria de 19 respiraciones por minuto, una $\mathrm{FiO} 2$ de 35\% y saturación de oxígeno que se mantiene por sobre el 90\%. Tiene una buena coloración cutánea, no hay signos de dificultad respiratoria. Hay secreciones abundantes en nariz y boca, y al lavado bronquial se obtienen secreciones hialinas y blanquecinas espesas. Presenta un Riesgo de aspiración relacionado a alimentación por sonda, depresión del reflejo nauseoso y tusígeno, deterioro de la deglución, disminución del nivel de conciencia, intubación endotraqueal, ERGE, retraso en el vaciado gástrico (7). La movilidad se encuentra ausente debido a la sedación, se encuentra hipotónico. Cuando se intenta suspender la sedación por primera vez, presenta una crisis convulsiva del tipo tónica; sin embargo, se baja la dosis del sedante y comienza a presentar movimientos repetitivos en las extremidades. Hay espasticidad marcada. Se diagnosticó el Deterioro de la movilidad física relacionado a deterioro cognitivo, neuromuscular y sensorioperceptivo, disminución del control muscular, disminución de la resistencia a medicamentos manifestado por enlentecimiento del movimiento, limitación de la amplitud de movimiento, movimientos espasmódicos (7). 
Reposo/sueño: No es posible valorar debido a la sedación, no hay distinción entre los estados de conciencia.

Cognitivo/perceptual: Hay reacción a la luz, pero no a estímulos táctiles. Las pupilas están mióticas, isocóricas, no hay llanto y se encuentra propenso a las crisis comiciales. Presenta movimientos repetitivos. En la tomografía cerebral se observa isquemia global, por lo cual se comunica a los padres que el daño neurológico es importante y permanente. Presentan un gran Riesgo de retraso en el desarrollo relacionado a crisis convulsiva, lesión cerebral (7).

Afrontamiento/tolerancia al estrés: Para la valoración del dolor se observó el comportamiento del paciente en respuestas al contacto físico o al realizar los procedimientos de enfermería. Se recomienda utilizar una la Escala de Dolor que sea capaz de valorar la expresión facial, llanto, respiración, movimiento corporal y estado de alerta. En ese caso, llevando a un diagnóstico de dolor agudo relacionado a agentes lesivos (punciones, lavado bronquial, aspiración) manifestado por cambios en la frecuencia cardiaca, conducta defensiva y gestos de protección (7).

Autopercepción/autoconcepto: Los padres se mantienen positivos en cuanto a la recuperación del paciente. Sin embargo reconocen sentirse cansados, preocupados y ansiosos.

Rol/relación: Vive con sus padres, abuelos y tíos, es el único en edad lactante en su familia. Sus padres reciben apoyo constante de familiares.

Sexualidad/reproducción: Los testículos se encuentran descendidos, el prepucio es retráctil, meato urinario con ubicación normal y ano permeable. Hay eritema y características propias de una infección micótica en zona perineal, así como dermatitis de pañal. Levantando del diagnóstico de Deterioro de la integridad cutánea relacionado a extremos de la edad, factores mecánicos, humedad, inmovilización física, deterioro de la sensibilidad y prominencias óseas manifestadas por la alteración de la superficie de la piel (7).

Afrontamiento/estrés: La conducta de los padres ante la hospitalización del bebé es positiva, pero sienten ansiedad constantemente, así como temor a tocar y mover al lactante debido al desconocimiento de su manejo.

Valores/creencias: Ambos padres son católicos, en la cuna hay imágenes religiosas y escapularios. Consideran la preservación de la vida como algo importante.

\section{PLAN DE CUIDADO Y RESULTADOS}

Ante los diagnósticos identificados fue posible establecer las mejores intervenciones y acompañar el desarrollo del paciente y su mejoría. Las intervenciones siguen como referencia las establecidas por el Nursing Interventions Classification (NIC) y para contrición de los resultados esperados el Nursing Outcomes Classification (NOC) $(8,9)$. Así fue posible presentar la construcción del plan de cuidados y los resultados obtenidos con la intervención de enfermería. 
El primero diagnóstico fue de Riesgo de glicemia inestable relacionado a alteración del estado mental, estado de salud física comprometido, al cual se logró llevar a un control del riesgo riguroso, y en unos días la glicemia se normaliza, se mantienen valores $>70 \mathrm{mg} / \mathrm{dl}$, (mejor expuesto en la tabla 1).

Tabla 1: Plan de cuidados para el diagnóstico: Riesgo de glicemia inestable relacionado a alteración del estado mental, estado de salud física comprometido.

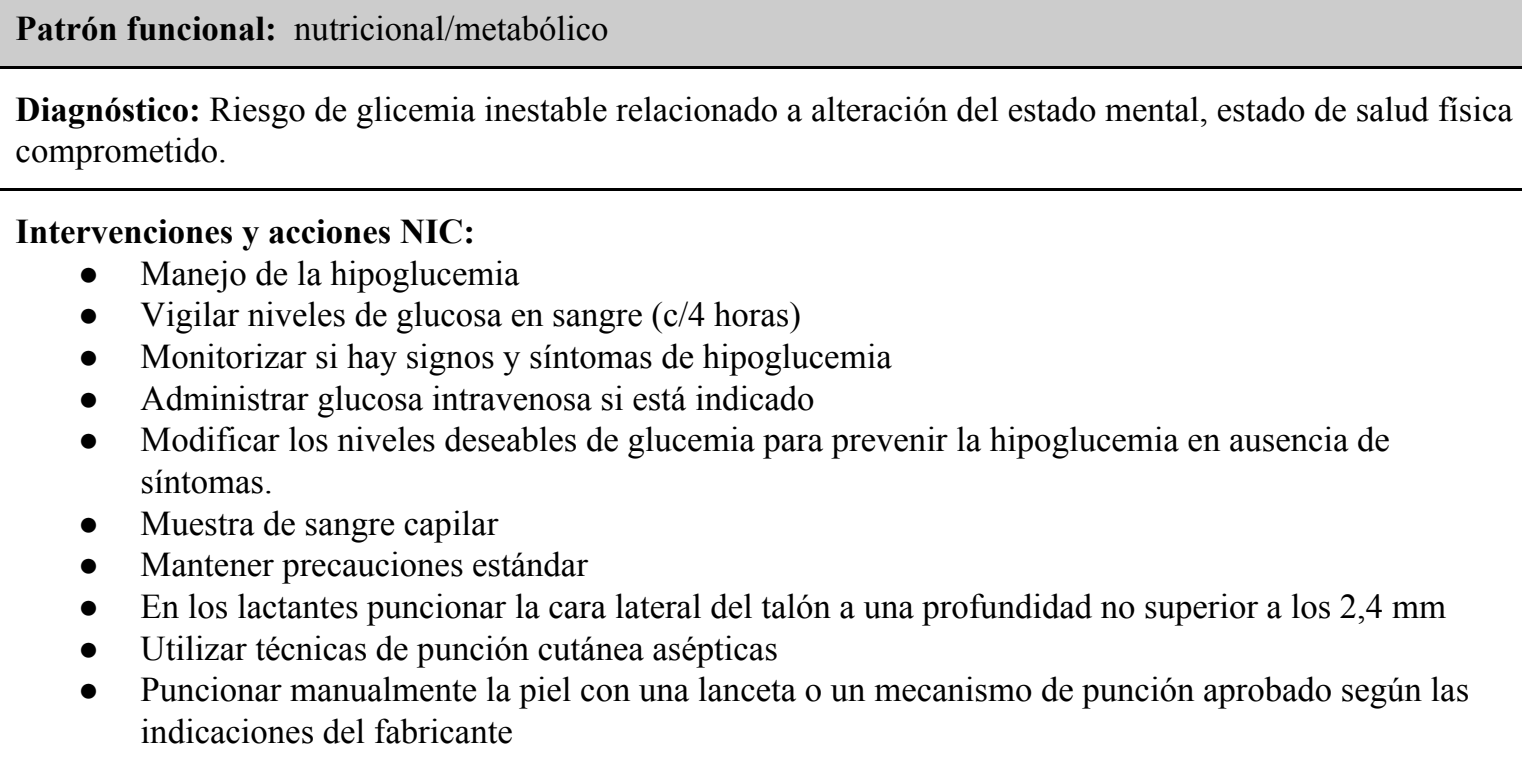

Diagnóstico: Riesgo de glicemia inestable relacionado a alteración del estado mental, estado de salud física comprometido.

\section{Intervenciones y acciones NIC:}

- Manejo de la hipoglucemia

- Vigilar niveles de glucosa en sangre (c/4 horas)

- Monitorizar si hay signos y síntomas de hipoglucemia

- Administrar glucosa intravenosa si está indicado

- Modificar los niveles deseables de glucemia para prevenir la hipoglucemia en ausencia de síntomas.

- Muestra de sangre capilar

- Mantener precauciones estándar

- En los lactantes puncionar la cara lateral del talón a una profundidad no superior a los 2,4 mm

- Utilizar técnicas de punción cutánea asépticas

- Puncionar manualmente la piel con una lanceta o un mecanismo de punción aprobado según las indicaciones del fabricante

Resultados NOC: Nivel de glucemia

\begin{tabular}{|c|c|c|c|c|c|}
\hline Puntuación & $\begin{array}{c}\text { Gravemente } \\
\text { comprometido }\end{array}$ & $\begin{array}{c}\text { Sustancialmente } \\
\text { comprometido }\end{array}$ & $\begin{array}{c}\text { Moderadamente } \\
\text { comprometido }\end{array}$ & $\begin{array}{c}\text { Levemente } \\
\text { comprometido }\end{array}$ & $\begin{array}{c}\text { No } \\
\text { comprometido }\end{array}$ \\
\hline $\begin{array}{c}\text { Concentració } \\
\text { n sanguínea } \\
\text { de glucosa }\end{array}$ & 1 & 2 & 3 & 4 & 5 \\
\hline
\end{tabular}

\begin{tabular}{|c|c|}
\hline \multicolumn{2}{|c|}{ Evaluaciones } \\
\hline Primera evaluación & Segunda evaluación \\
\hline $2 / 5$ & $5 / 5$ \\
\hline
\end{tabular}

Fuente: Elaboración propia (2018)

En el diagnóstico de deterioro de la integridad tisular relacionado a daños en la superficie ocular, efectos secundarios del tratamiento, factores ambientales, lesiones neurológicas con pérdida de reflejo sensorial, ventilación mecánica manifestado por lesión tisular (conjuntiva), se empezó el tratamiento con cloranfenicol. Al no obtener resultados, pasa por una valoración de la oftalmóloga, quien le diagnostica quemosis. Se cambió el tratamiento para prednisolona e hipromelosa oftálmicas, que logra desaparecer en pocos días después de iniciar el nuevo tratamiento. Esto se puede observar las intervenciones en la tabla 2. 
Tabla 2. Plan de cuidados para el Diagnóstico: Deterioro de la integridad tisular relacionado a daños en la superficie ocular.

Patrón funcional: nutricional/metabólico

Diagnóstico: Deterioro de la integridad tisular relacionado a daños en la superficie ocular, efectos secundarios del tratamiento, factores ambientales, lesiones neurológicas con pérdida de reflejo sensorial, ventilación mecánica manifestado por lesión tisular (conjuntiva).

Intervenciones y acciones NIC

Cuidado de los ojos

- Observar enrojecimiento, exudado, ulceración

- Aplicar colirio lubricante

- Mantener párpado cerrado

Administración de medicación: oftálmica

- Seguir las reglas de administración

- Instilar medicación en saco conjuntival usando técnica aséptica, previa limpieza de la zona eliminando secreciones

- Lavado de manos

Resultados NOC: Severidad del ojo seco

\begin{tabular}{|c|c|c|c|c|c|}
\hline Puntuación & Grave & Sustancial & Moderado & Leve & Ninguno \\
\hline $\begin{array}{c}\text { Disminución de la } \\
\text { producción de } \\
\text { lágrimas }\end{array}$ & 1 & 2 & 3 & 4 & 5 \\
\hline $\begin{array}{c}\text { Cierre palpebral } \\
\text { incompleto }\end{array}$ & 1 & 2 & 3 & 4 & 5 \\
\hline $\begin{array}{c}\text { Enrojecimiento de } \\
\text { la conjuntiva }\end{array}$ & 1 & 2 & 3 & 4 & 5 \\
\hline $\begin{array}{c}\text { Exceso de } \\
\text { secreciones }\end{array}$ & 1 & 2 & 3 & 4 & 5 \\
\hline
\end{tabular}

\begin{tabular}{|c|c|}
\hline \multicolumn{2}{|c|}{ Evaluaciones } \\
\hline Primera evaluación & Segunda evaluación \\
\hline $7 / 20$ & $20 / 20$ \\
\hline
\end{tabular}

Fuente: Elaboración Propia (2018)

Para el exceso de volumen de líquido relacionado a compromiso de los mecanismos reguladores manifestado por aportes superiores a las pérdidas, hipertensión pulmonar moderada, oliguria y edema, se logra un balance hídrico más equilibrado. Como resultado después de la intervención fue posible mejorar los edemas antes presentados, como demuestra la tabla 3. 
Tabla 3. Plan de cuidado para el diagnóstico del exceso de volumen de líquido.

\begin{tabular}{|c|c|c|c|c|c|}
\hline \multicolumn{6}{|c|}{$\begin{array}{l}\text { Patrón funcional: nutricional/metabólico } \\
\text { Diagnóstico: Exceso de volumen de líquido relacio }\end{array}$} \\
\hline \multicolumn{6}{|c|}{$\begin{array}{l}\text { Intervenciones y acciones NIC } \\
\text { Manejo de líquidos } \\
\text { - Pesar pañales } \\
\text { - } \text { Realizar registro preciso de entradas y salidas } \\
\text { - Monitolar resultados de laboratorio relevantes (+BUN o-Hto) } \\
\text { - Evaluar ubicación y extensión de edema } \\
\text { Monitorización de líquidos } \\
\text { - Determinar cantidad y tipo de líquidos de ingesta y hábitos de evacuación } \\
\text { - } \text { Explorar relleno capilar y turgencia } \\
\text { - Monitorizar peso }\end{array}$} \\
\hline \multicolumn{6}{|c|}{ Resultados NOC: Equilibrio hídrico } \\
\hline \begin{tabular}{|l} 
Puntuación \\
Indicadores
\end{tabular} & $\begin{array}{l}\text { Gravemente } \\
\text { comprometido }\end{array}$ & $\begin{array}{l}\text { Sustancialmente } \\
\text { comprometido }\end{array}$ & $\begin{array}{l}\text { Moderadamente } \\
\text { comprometido }\end{array}$ & $\begin{array}{l}\text { Levemente } \\
\text { comprometido }\end{array}$ & $\begin{array}{c}\text { No } \\
\text { comprometido }\end{array}$ \\
\hline $\begin{array}{c}\text { Entradas y } \\
\text { salidas } \\
\text { diarias } \\
\text { equilibradas } \\
\end{array}$ & 1 & 2 & 3 & 4 & 5 \\
\hline $\begin{array}{c}\text { Edema } \\
\text { periférico }\end{array}$ & 1 & 2 & 3 & 4 & 5 \\
\hline $\begin{array}{l}\text { Edema } \\
\text { palpebral }\end{array}$ & 1 & 2 & 3 & 4 & 5 \\
\hline $\begin{array}{l}\text { Cantidad de } \\
\text { orina }\end{array}$ & 1 & 2 & 3 & 4 & 5 \\
\hline \multirow[t]{4}{*}{ Hematocrito } & 1 & 2 & 3 & 4 & 5 \\
\hline & \multicolumn{4}{|c|}{ Evaluaciones } & \\
\hline & \multirow{2}{*}{\multicolumn{2}{|c|}{$\frac{\text { Primera evaluación }}{13 / 25}$}} & \multicolumn{2}{|c|}{ Segunda evaluación } & \\
\hline & & & & & \\
\hline
\end{tabular}

Fuente: Elaboración Propia (2018)

El paciente presentó un gran riesgo de sangrado relacionado a coagulopatías esenciales. Con las intervenciones fue posible apenas notar poca diferencia, como demuestra la tabla 4. En la ruta de desarrollo el paciente fue sujeto a una transfusión de 57 mililitros de plasma fresco congelado y dos paquetes globulares de 57 mililitros cada uno. 
Tabla 4. Plan de cuidado para el diagnóstico de riesgo de sangrado relacionado a coagulopatías esenciales.

\begin{tabular}{|c|c|c|c|c|c|}
\hline \multicolumn{6}{|c|}{ Patrón funcional: actividad/ejercicio } \\
\hline \multicolumn{6}{|c|}{ Diagnóstico: Riesgo de sangrado relacionado a coagulopatías esenciales. } \\
\hline \multicolumn{6}{|c|}{$\begin{array}{l}\text { Intervenciones y acciones } \\
\text { Administración de hemoderivados } \\
\text { - Verificar indicaciones } \\
\text { - Verificar el hemoderivado (grupo sanguíneo, grupo Rh, número de unidad) } \\
\text { - Registrar signos y vitales antes, durante y después del procedimiento } \\
\text { - Regular el flujo } \\
\text { - Registra duración del procedimiento } \\
\text { Registrar volumen }\end{array}$} \\
\hline \multicolumn{6}{|c|}{ Resultados NOC: Coagulación sanguínea } \\
\hline \begin{tabular}{|l} 
Puntuación \\
Indicadores \\
\end{tabular} & $\begin{array}{l}\text { Desviación } \\
\text { grave }\end{array}$ & $\begin{array}{c}\text { Desviación } \\
\text { sustancial }\end{array}$ & $\begin{array}{c}\text { Desviación } \\
\text { moderada }\end{array}$ & $\begin{array}{l}\text { Desviación } \\
\text { leve }\end{array}$ & $\begin{array}{c}\text { Sin } \\
\text { desviación }\end{array}$ \\
\hline Petequias & 1 & 2 & 3 & 4 & 5 \\
\hline TPT & 1 & 2 & 3 & 4 & 5 \\
\hline $\begin{array}{l}\text { Concentración } \\
\text { plasmática de } \\
\text { fibribógeno }\end{array}$ & 1 & 2 & 3 & 4 & 5 \\
\hline $\begin{array}{c}\text { Concentración de } \\
\text { plaquetas }\end{array}$ & 1 & 2 & 3 & 4 & 5 \\
\hline Hematocrito & 1 & 2 & 3 & 4 & 5 \\
\hline \multirow[t]{4}{*}{ Hemoglobina } & 1 & 2 & 3 & 4 & 5 \\
\hline & \multicolumn{4}{|c|}{ Evaluaciones } & \\
\hline & rimera evalua & & Segunda & luación & \\
\hline & $20 / 30$ & & & & \\
\hline
\end{tabular}

Fuente: Elaboración Propia (2018)

Para el Riesgo de aspiración relacionado a alimentación por sonda, depresión del reflejo nauseoso y tusígeno, deterioro de la deglución, disminución del nivel de conciencia, intubación endotraqueal, ERGE, retraso en el vaciado gástrico, fue desarrollado el plan de intervención presentado en la tabla 5. Fue posible a presentar una buena evolución, y disminuir el riesgo de aspiración parcialmente. Es importante resaltar que al momento de la segunda evaluación, se suspendió el ayuno y se le comenzó a alimentar con 40 mililitros de fórmula para lactantes por sonda orogástrica. 
Tabla 5. Plan de cuidado para el diagnóstico de riesgo de aspiración.

\begin{tabular}{|c|c|c|c|c|c|}
\hline \multicolumn{6}{|c|}{$\begin{array}{l}\text { Diagnóstico: Riesgo de aspiración relacionado a alimentación por sonda, depresión del reflejo nauseoso y } \\
\text { tusígeno, deterioro de la deglución, disminución del nivel de conciencia, intubación endotraqueal, ERGE, } \\
\text { retraso en el vaciado gástrico. }\end{array}$} \\
\hline $\begin{array}{c}\text { Intervenciones y acci } \\
\text { Aspiración de la vía aé } \\
\text { - } \text { Determinar } \\
\text { - } \text { Auscultar a } \\
\text { - Selecciona } \\
\text { - } \text { Monitoriza } \\
\text { Precaución para evitar } \\
\text { - } \text { Minimizar } \\
\text { - Cabecera e } \\
\text { - } \text { Proporcion } \\
\text { - Comproba } \\
\text { - Comproba } \\
\end{array}$ & $\begin{array}{l}\text { eas } \\
\text { lecesidad de as } \\
\text { tes y después } \\
\text { ar antes y desp } \\
\text { sonda adecuada } \\
\text { oxigenación y } \\
\text { observar color, } \\
\text { aspiración } \\
\text { l uso de sedante } \\
\text { vada luego de } \\
\text { alimentación } \\
\text { colocación de la } \\
\text { esiduo de sond } \\
\text { méticos }\end{array}$ & $\begin{array}{l}\text { iración } \\
\text { és de cada aspir } \\
\text { antado hemodiná } \\
\text { antidad y consi }\end{array}$ & $\begin{array}{l}\text { ción } \\
\text { hico } \\
\text { encia de las sec }\end{array}$ & eciones & \\
\hline \multicolumn{6}{|c|}{$\begin{array}{c}\text { • Uso de antieméticos } \\
\text { Resultados NOC: Estado respiratorio: permeabilidad de las vías respiratorias }\end{array}$} \\
\hline $\begin{array}{l}\text { Puntuación } \\
\text { Indicadores }\end{array}$ & $\begin{array}{l}\text { Desviación } \\
\text { grave del } \\
\text { rango normal }\end{array}$ & $\begin{array}{l}\text { Desviación } \\
\text { sustancial del } \\
\text { rango normal }\end{array}$ & $\begin{array}{l}\text { Desviación } \\
\text { moderada del } \\
\text { rango normal }\end{array}$ & $\begin{array}{l}\text { Desviación } \\
\text { leve del } \\
\text { rango normal }\end{array}$ & $\begin{array}{l}\text { Sin } \\
\text { desviación } \\
\text { del rango } \\
\text { normal }\end{array}$ \\
\hline $\begin{array}{l}\text { Capacidad de } \\
\text { eliminar } \\
\text { secreciones } \\
\end{array}$ & 1 & 2 & 3 & 4 & 5 \\
\hline \multirow[t]{4}{*}{$\begin{array}{l}\text { Acumulación de } \\
\text { esputo }\end{array}$} & 1 & 2 & 3 & 4 & 5 \\
\hline & \multicolumn{4}{|c|}{ Evaluaciones } & \\
\hline & \multicolumn{2}{|c|}{ Primera evaluación } & \multicolumn{2}{|c|}{ Segunda evaluación } & \\
\hline & \multicolumn{2}{|c|}{$3 / 10$} & \multicolumn{2}{|c|}{$6 / 10$} & \\
\hline
\end{tabular}

Fuente: Elaboración Propia (2018)

Para el diagnóstico de deterioro de la movilidad física, fue posible mejorar su desempeño articular, urinario y reduciendo el riesgo de ulcera por presión, como se puede observar la evolución de este cuidado en la tabla 6. 
Tabla 6. Plan de cuidado para el diagnóstico de deterioro de la movilidad física.

\section{Patrón funcional: actividad/ejercicio}

Diagnóstico: Deterioro de la movilidad física relacionado a deterioro cognitivo, deterioro neuromuscular, deterioro sensorioperceptivo, disminución del control muscular, disminución de la resistencia y medicamentos manifestado por enlentecimiento del movimiento, limitación de la amplitud de movimiento, movimientos espasmódicos

\section{Intervenciones $\mathbf{y}$ acciones}

Terapia de ejercicios: movilidad articular

- Determinar limitaciones del movimiento

- Colaborar con fisioterapia en el desarrollo y ejecución de un programa de ejercicios

- Enseñar a la familia a realizar de forma sistemática los ejercicios

Resultados NOC: Consecuencias de la inmovilidad: fisiológicas

\begin{tabular}{|c|c|c|c|c|c|}
\hline Puntuación & Grave & Sustancial & Moderado & Leve & Ninguno \\
\hline $\begin{array}{c}\text { Undicadores } \\
\text { úceras por } \\
\text { presión }\end{array}$ & 1 & 2 & 3 & 4 & 5 \\
\hline $\begin{array}{c}\text { Retención } \\
\text { urinaria }\end{array}$ & 1 & 2 & 3 & 4 & 5 \\
\hline $\begin{array}{c}\text { Movimiento } \\
\text { articular }\end{array}$ & 1 & 2 & 3 & 4 & 5 \\
\hline
\end{tabular}

\begin{tabular}{|c|c|}
\hline \multicolumn{2}{|c|}{ Evaluaciones } \\
\hline Primera evaluación & Segunda evaluación \\
\hline $7 / 15$ & $12 / 15$ \\
\hline
\end{tabular}

Fuente: Elaboración Propia (2018)

Para el diagnóstico de riesgo de retraso en el desarrollo relacionado a crisis convulsiva y lesión cerebral, fue posible planear como prevenir y manejar en los momentos de convulsiones, como se expone en la tabla 7. 
Tabla 7. Plan de cuidado para el diagnóstico de riesgo de retraso en el desarrollo.

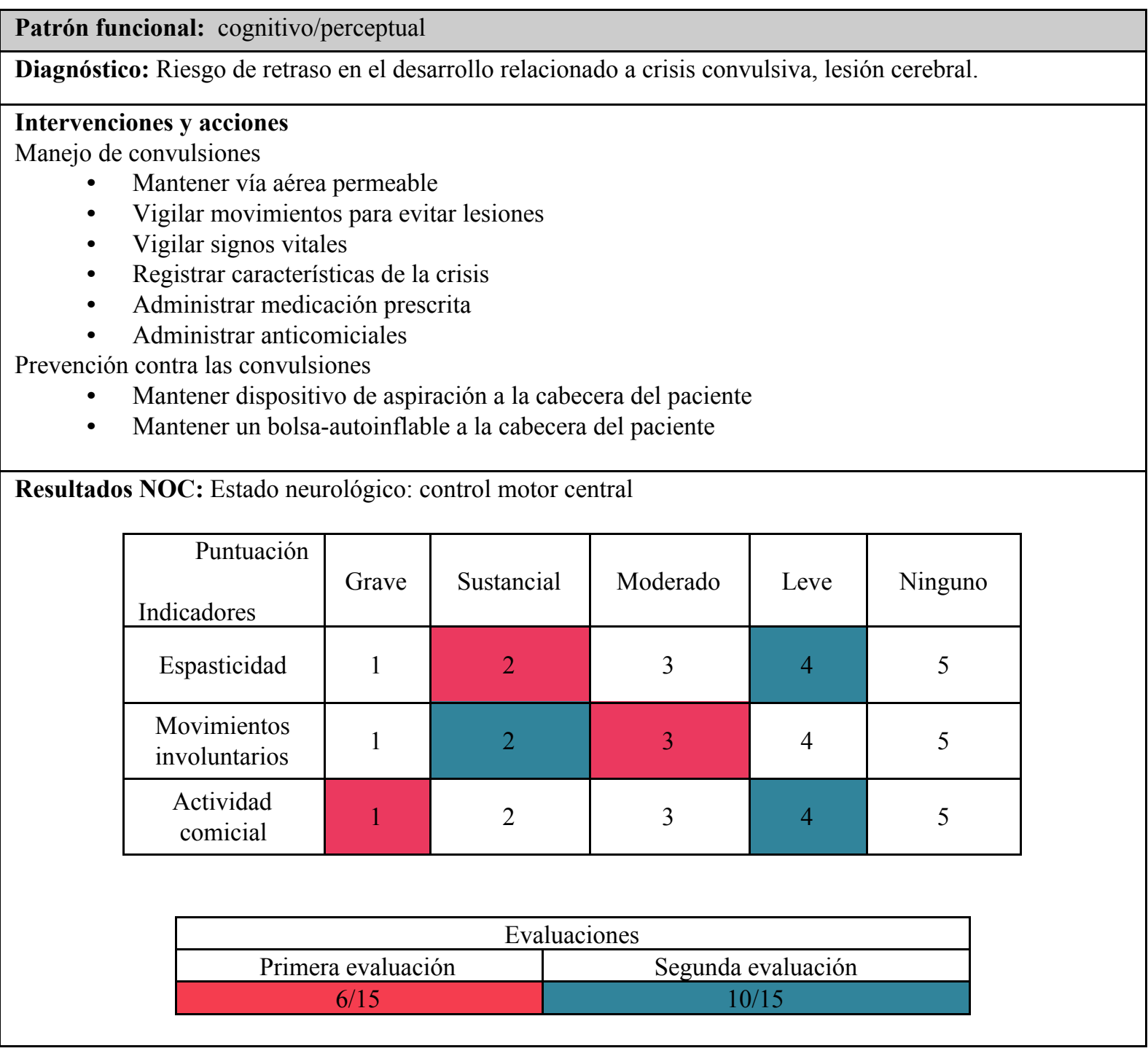

Fuente: Elaboración Propia (2018)

Para el diagnóstico de deterioro de la integridad cutánea, se planeó una intervención de cuidados enfocado en evitar las lesiones por presión, como se presenta en la tabla 8 . Presentó gran evolución después de la aplicación de pantenol y miconazol tópicos en zona genital. 
Tabla 8. Plan de cuidado para el diagnóstico de deterioro de la integridad cutánea.

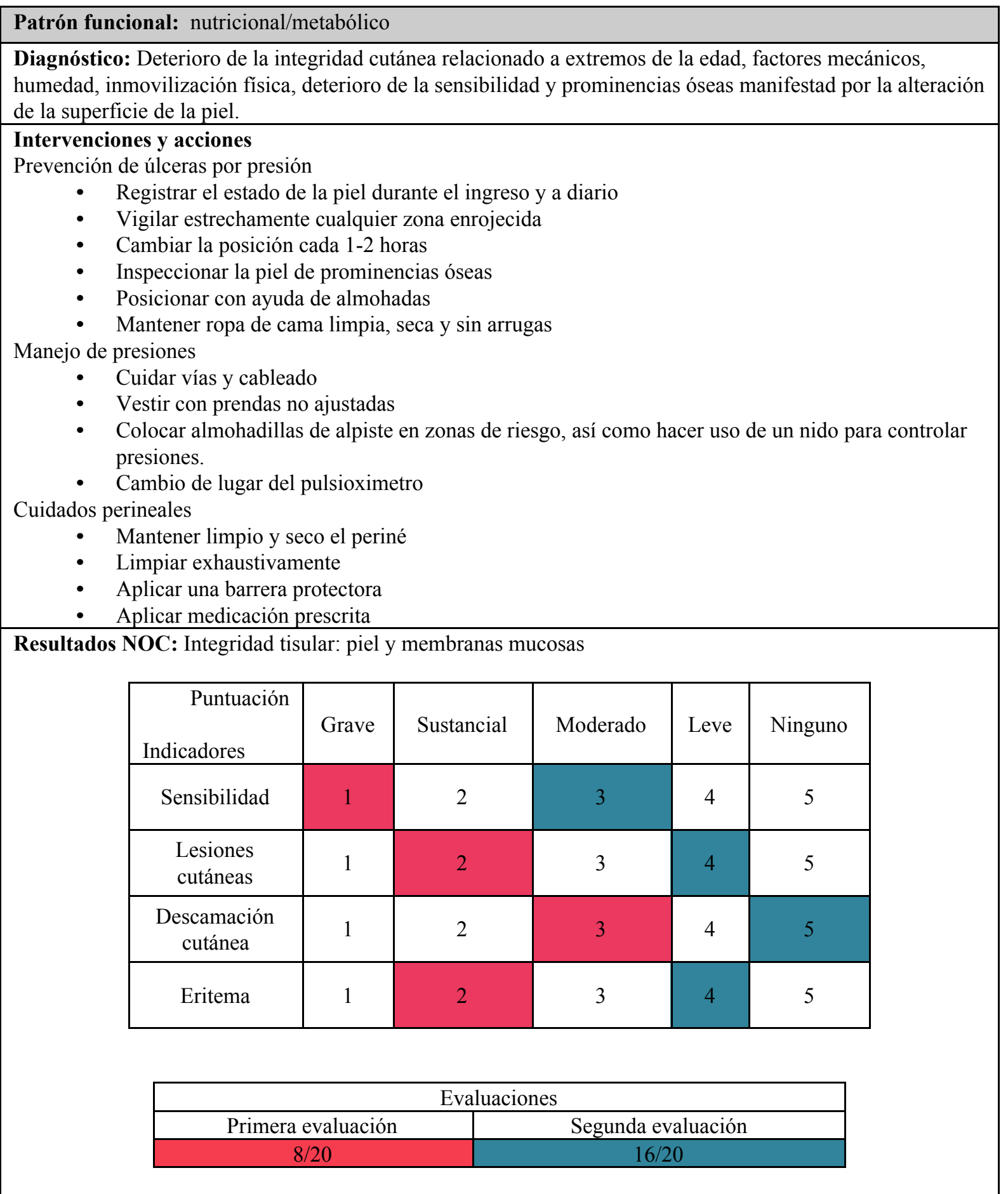

Fuente: Elaboración Propia (2018)

En el diagnóstico de dolor agudo relacionado a agentes lesivos (punciones, lavado bronquial, aspiración) manifestado por cambios en la frecuencia cardiaca, conducta defensiva y gestos de protección, es posible visualizar las intervenciones realizadas en la tabla 9. Con las intervenciones se logra una evolución satisfactoria presentando una reducción de las conductas defensivas y gestos de protección. 
Tabla 9. Plan de cuidado para el diagnóstico de dolor agudo relacionado a agentes lesivos.

\begin{tabular}{|c|c|c|c|c|c|}
\hline \multicolumn{6}{|c|}{ Patrón funcional: afrontamiento/tolerancia al estrés } \\
\hline \multicolumn{6}{|c|}{$\begin{array}{l}\text { Diagnóstico: Dolor agudo relacionado a agentes lesivos (punciones, lavado bronquial, aspiración) } \\
\text { manifestado por cambios en la frecuencia cardiaca, conducta defensiva y gestos de protección. }\end{array}$} \\
\hline \multicolumn{6}{|c|}{$\begin{array}{l}\text { Intervenciones y acciones } \\
\text { Contacto } \\
\text { - Animar a los padres a que masajeen al lactante } \\
\text { - Animar a los padres a que toquen al niño enfermo } \\
\text { - Envolver al lactante en una manta } \\
\text { - Evocar nido (anidamiento) } \\
\text { - Lavado el efecto del contacto } \\
\end{array}$} \\
\hline \multicolumn{6}{|c|}{ Resultados NOC: Estado de comodidad física } \\
\hline $\begin{array}{l}\text { Puntuación } \\
\text { Indicadores }\end{array}$ & $\begin{array}{l}\text { Gravemente } \\
\text { comprometido }\end{array}$ & $\begin{array}{l}\text { Sustancialmente } \\
\text { comprometido }\end{array}$ & $\begin{array}{l}\text { Moderadamente } \\
\text { comprometido }\end{array}$ & $\begin{array}{l}\text { Levemente } \\
\text { comprometido }\end{array}$ & $\begin{array}{l}\text { No } \\
\text { comprometido }\end{array}$ \\
\hline $\begin{array}{l}\text { Bienestar } \\
\text { físico }\end{array}$ & 1 & 2 & 3 & 4 & 5 \\
\hline \multirow[t]{4}{*}{$\begin{array}{l}\text { Relajación } \\
\text { muscular }\end{array}$} & 1 & 2 & 3 & 4 & 5 \\
\hline & \multicolumn{4}{|c|}{ Evaluaciones } & \\
\hline & \multicolumn{2}{|c|}{ Primera evaluación } & \multicolumn{2}{|c|}{ Segunda evaluación } & \\
\hline & \multicolumn{2}{|c|}{$4 / 10$} & \multicolumn{2}{|c|}{$9 / 10$} & \\
\hline
\end{tabular}

Fuente: Elaboración Propia (2018)

El tratamiento de pacientes con AP y EHI necesita cuidados integrales para los servicios prestados, que se inicia en la sala de parto y sigue hasta la unidad de terapia intensiva. La hipotermia controlada de forma segura es la mejor estrategia para reducir la mortalidad relacionada con la EHI; la creación de protocolos permite la introducción de la atención a los pacientes (10). El tratamiento por medio de hipotermia asociado a las intervenciones de enfermería presentadas en este estudio, que obtuvieron resultados positivos, compone un protocolo relevante para una asistencia integral para los pacientes.

\section{CONSIDERACIÓN FINAL}

La aplicación del proceso de enfermería durante el servicio social posibilitó el desarrollo de una asistencia basada en evidencia bajo el uso de las herramientas NANDA, NIC y NOC, colaborando para la formación de profesionales capaces de implementar una asistencia de calidad. Lo ocurrido aquí es considerado como un caso esporádico, debido a una solicitud de alta voluntaria en el medio del proceso de mejora del cuadro clínico, generando complicaciones y agravando la situación clínica. El relato de este caso evidencia la importancia de la enfermería en las intervenciones en forma precoz, en conjunto con un equipo multidisciplinario, adecuar la asistencia y posibilitar una calidad de vida al paciente y miembros familiares. 


\section{REFERENCIAS BIBLIOGRÁFICAS}

1. Blasina F. Protección del recién nacido frente a la asfixia perinatal : perspectivas de un largo camino. Arch Pediatr Urug. 2016;87(3):195-7.

2. Lemus-Varela M, Sola A, Golombek S, Baquero H, Dávila-Aliaga C, Fariña D, et al. Recomendaciones terapeuticas del VII Consenso Clinico de SIBEN para la encefalopatia hipoxico-isquemica neonatal. Neoreviews [Internet]. 2016; 17 (9): 554-67.

http://neoreviews.aappublications.org/cgi/doi/10.1542/neo.17-9-e554

3. Balushi A, López M, Wintermark P. Impacto de la ventilación en el desarrollo de daño cerebral en recién nacidos con asfixia, tratados con hipotermia. Anest en México. 2017;2 9(1): 30-40.

4. Grupo de Trabajo Hipotermia Terapéutica C de EF-N (CEFEN). Recomendación para el tratamiento con hipotermia en recién nacidos con encefalopatía hipóxicoisquémica. Arch Argent Pediatr [Internet]. 2017;115 (3:s):38-52. Disponible en: http://www.sap.org.ar/docs/publicaciones/archivosarg/2017/v115n3a26s.pdf

5. Rivera M, Lara N, Baró T. Asfixia al nacer: factores de riesgo materno y su repercusión en la mortalidad neonatal. Rev Ide Inf Científica. 2017; 96 (6): 1143-52.

6. Barbosa M de LG, Castro CMMB de, Telles MVL, Belarmino ALB, Parente LLT, Mendonça A, et al. Prevalência de Hipóxia Perinatal em Gemelares da Maternidade Pública em Juazeiro do Norte-CE. Id Line Rev Psic. 2016;10 (31): 202-23.

7. Riesgo-prendes L, Salamanca Matta A, Monterrey Gutiérrez P, Bermúdez Hernández P, Vélez J. Hipoxia perinatal en el Hospital Mederi de Bogotá: comportamiento en los años 2007 a 2011. Rev Salud Pública. 2017; 19 (3): 332-9.

8. Segur P, Morero J, Oliveira C. Assistência de Enfermagem ao recém-nascido com Sindrome do Desconforto Respiratório. Rev UNINGÁ. 2019;56 (S2): 141-59.

9. Hernández EES. Síndrome Rusell Silver. Presentación de caso. Rev Méd Electrón [Internet]. $2018 ; 40(3):$ 784-9.

10. Silvera F, Gesuele JP, Oca RM, Vidal G, Martínez V, Lucas L, et al. Neuroprotección en pacientes con asfixia perinatal. Arch Pediatr Urug. 2016 ;87(3): 221-33. 